Combining gait optimization with passive system to increase the energy efficiency of a humanoid robot walking movement

Ana I. Pereira, José Lima, and Paulo Costa

Citation: AIP Conference Proceedings 1648, 140002 (2015); doi: 10.1063/1.4912422

View online: https://doi.org/10.1063/1.4912422

View Table of Contents: http://aip.scitation.org/toc/apc/1648/1

Published by the American Institute of Physics 


\title{
Combining Gait Optimization with Passive System to Increase the Energy Efficiency of a Humanoid Robot Walking Movement
}

\author{
Ana I. Pereira*,†, José Lima ${ }^{*, * *}$ and Paulo Costa ${ }^{\ddagger, * *}$ \\ *Polytechnic Institute of Bragança, Portugal \\ ${ }^{\dagger}$ ALGORITMI,University of Minho, Portugal \\ **INESC TEC (formerly INESC Porto) Porto, Portugal \\ ${ }^{\ddagger}$ Faculty of Engineering, University of Porto, Portugal
}

\begin{abstract}
There are several approaches to create the Humanoid robot gait planning. This problem presents a large number of unknown parameters that should be found to make the humanoid robot to walk. Optimization in simulation models can be used to find the gait based on several criteria such as energy minimization, acceleration, step length among the others. The energy consumption can also be reduced with elastic elements coupled to each joint. The presented paper addresses an optimization method, the Stretched Simulated Annealing, that runs in an accurate and stable simulation model to find the optimal gait combined with elastic elements. Final results demonstrate that optimization is a valid gait planning technique.
\end{abstract}

Keywords: Humanoid Gait. Simulated annealing. Optimization.

PACS: $02.60 . P n$

\section{INTRODUCTION}

Recently, humanoid robots have been enjoying great popularity and are now used as a research tool in many groups worldwide. They are a challenging task due to its hard control. Moreover, gait generation and optimization still remain a challenge for such a high-order highly-coupled nonlinear dynamical system [1]. The humanoid robot gait planning presents a large number of unknown parameters that should be found to make the humanoid robot to walk. It can be approached in two ways: the online (done in real-time that requires high computational effort) and the offline gait generation methods. This offline approach, brings some advantages such as the ability to use complex algorithms to find an optimal solution.

The optimization of the humanoid robot gait is a good area to apply optimization methods over the simulated robot. It is prudent to avoid time-consuming optimization runs that wear out the robot hardware. Several approaches have been presented that aim at optimizing properties such as speed $[2,3]$ or torso stability $[4,5]$ of a humanoid's walk.

In order to generate walking patterns for different locomotion kinematics, the common way of most existing approaches is to precompute reference trajectories [6].On the one hand, the gait can also be planned having in mind the energy consumption: the energy/distance ratio can be observed and minimized. On the other hand, a passive system that coupled to the humanoid robot joints allows to save energy. The passive system optimal characteristics depend on each joint desired trajectory. These characteristics can be found by an optimization method. For this purpose a realistic model for the simulator (SimTwo [7]) was developed working as a base to compute the combined elastic elements (k and offset) constraints and gait parameters. SimTwo is a physical robot simulator that is capable to simulate userdefined robots in three-dimensional space since it includes a physical model based on rigid body dynamics (the $O D E$ - Open Dynamics Engine [8]).

Previous authors works address these optimization ways separately: optimization of gait $([9,10])$ and elastic elements parameters for each joint. This paper combines both optimization problems increasing the dimension and complexity of the solutions. The main optimization technique is implemented resorting to the Stretched Simulated Annealing method. It is a generic probabilistic method for the global optimization problem. It tries to find the global minimum of a given function in a large search space (as it happens in humanoid gait planning and elastic elements parameters) and can escape from local minima. Besides, there must be used an optimizing method because it is unthinkable to perform a complete search (using brute force) once the search space is huge (i.e. dimension 120). There are similar works that addresses gait optimization [11] but the union of gait with elastic elements is an innovative 
approach.

The paper is organized as follows: Section 2 presents the developed simulation model (SimTwo) with its parameters. Then, in Section 3, the optimization techniques based on Stretched Simulated Annealing is addressed. Section 4 presents a discussion of results and finally, the last section concludes this work and gives some future work on this topic.

\section{SIMULATION MODEL}

Actually, manufacturers of robots as well as specialized companies on modeling and simulation provide accurate simulators. It allows to create, develop and test robots algorithms avoiding damages, decreasing costs and speeding up the control of robots. The basis of simulation is composed by models (actuators, sensors and environment) and physics engine responsible of the dynamic behaviour (computed by the ODE - Open Dynamics Engine [8], which is a free library for simulating rigid body dynamics).

The used simulator, SimTwo, is a generic simulator that allows to create personalized robots and user can access the low level behaviour, such as dynamical model, friction model, servomotor model and sensors model in a way that developed code can be easily transferred to the real robot. Moreover, a stable version is available and capable of compute complex models that allows to simulate a robot with a high number of joints in a chain architecture without instability and noise (previously presented in [12]). SimTwo also allows to run simulations faster than real time: a very useful property to accelerate the research task. A snapshot of the developed simulator is presented in Figure 1.

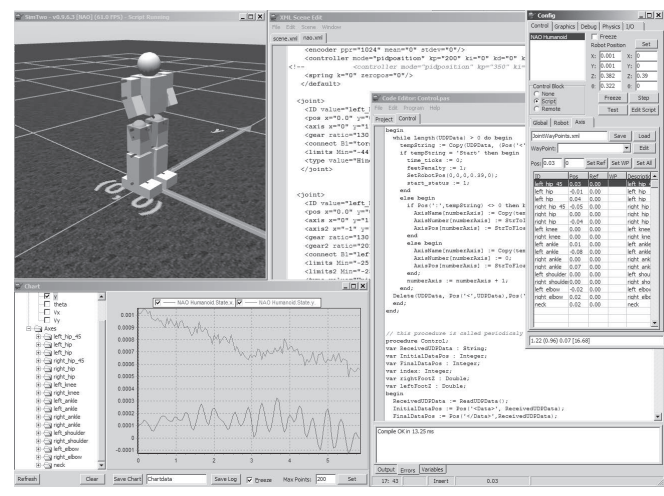

FIGURE 1. SimTwo simulator environment.

The dynamics realism in SimTwo is obtained by decomposing a robot in rigid bodies and electric motors (joints). Each body behaviour is numerically simulated using its physical characteristics such as shape, mass and moments of inertia, surface friction and elasticity. It is also possible to define standard joints such as socket, hinge and slider which can be coupled with an actuator or a sensor. Previous works validates the realism of the SimTwo simulator [13].

The gait-planning is one of the fundamental problems in humanoid mobile robots. The problem of gait planning for humanoid robots is fundamentally different from the path planning for wheeled robots due to the inherent characteristics of legged locomotion. The main challenge of gait planning is to find constraint functions and their associated gait parameters. However, finding repeatable gait when the constraint equations involve higher order differential equations still remained unsolved [14]. There are the online and the offline generation methods [14]. The first one, should be done in real time and requires a high computational effort. On the other hand, a popular way to solve this problem is to resort to offline optimization techniques. This paper applies the model parameters and actuator inputs that lead to fully open-loop stable walking motions based on a simplified robot model to increase stability (Figure 2).

Simulator receives the gait parameters and elastic elements constants from Matlab, runs it and returns the humanoid energy consumption through UDP network packet for each optimization iteration.

The next section briefly describes the main ideas behind the used optimization method. 

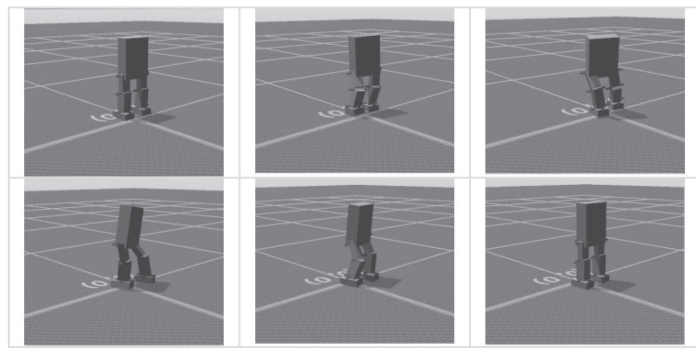

FIGURE 2. SimTwo Humanoid Simplified model walking.

\section{OPTIMIZATION METHODS}

A well known optimization method was used in the humanoid gait planning: the Stretched Simulated Annealing.

The SSA method belongs to the class of the multilocal optimization methods. The SSA method solves, in each iteration, a global optimization problem using the simulated annealing (SA) algorithm. The SA is a point-to-point stochastic algorithm that does not require derivative information and is able to guarantee convergence to a global solution with probability one. In each iteration of the SSA method, the global problem is transformed using a function stretching technique [15].

\section{NUMERICAL RESULTS}

The numerical results were obtained using a Inter Core i7-2600 CPU $3.4 \mathrm{GHz}$ with $8.0 \mathrm{~GB}$ of RAM.

For the optimization procedure, the input data is a matrix with dimension $12 \times 10$. The initial approximation was obtained through empirical analysis. The matrix $x_{0}$ presents the initial gait for 12 joints (lines) and for 10 time instants (columns).

The upper and lower limits are defined using $x_{0}$, as $l=x_{0}-\alpha 1_{10}$ and $u=x_{0}+\alpha 1_{10}$, where $1_{10} \in \mathbb{R}^{12 \times 10}$ is a matrix with coefficient one in all positions. The energy consumed to power the motors for all the joints of the humanoid robot when considered the input variable $x_{0}$ is $f\left(x_{0}\right)=1013.5 \mathrm{Joules} / \mathrm{m}$.

In SSA method the parameters $\delta_{1}, \delta_{2}, \kappa$ and $\alpha$ were fixed as $10^{2}, 1,10^{-3}$ and 2 , respectively. The maximum number of function evaluations was set to 5000 and the maximum number of iterations was set as 5000. Since SSA method is stochastic, the problem was solved five times and the numerical results presented in the following table are average values over the runs. The Table 1 presents for each run the optimum value obtained, $F\left(x^{*}\right)$, and the average of the optimum values in each run, $F_{A}\left(x^{*}\right)$.

TABLE 1. Numerical results using

\begin{tabular}{lcc}
\multicolumn{3}{l}{ SSA method. } \\
\hline Runs & $F\left(x^{*}\right)$ & $F_{A}\left(x^{*}\right)$ \\
\hline 1 & 699.5 & 777.9 \\
2 & 700.3 & 747.4 \\
3 & 714.5 & 790.8 \\
4 & 706.8 & 754.7 \\
5 & 726.0 & 778.5 \\
\hline
\end{tabular}

The Figure 3 presents the evolution of the optimum value during the best run.

SSA method was able to improve the initial solution $69 \%$. The minimum value was only obtained in the first run of SSA method. The average minima values of all runs was 709.4. It is possible to see that the minimum values are not equal in all runs, this can be explained by the fact that the optimization method is stochastic procedure.

\section{CONCLUSIONS AND FUTURE WORK}

In this paper a Stretched simulated annealing application for creating optimized energy of humanoid robots was presented. For that purpose, tests were conducted on the personalized simulator SimTwo, a robot simulator that 


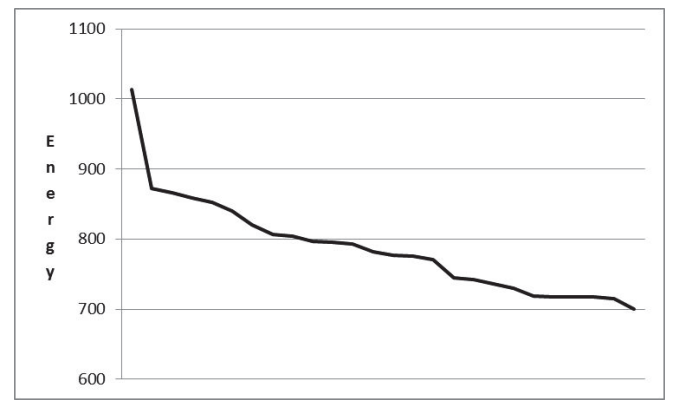

FIGURE 3. Evolution optimum values during the best run.

is capable of simulating user-defined robots in three-dimensional space with physical model based on rigid body dynamics. With 5000 functions evaluations the SSA algorithm identifies the best solution for the optimization problem improving 69\% the initial solution. Once this approach is validated, the implementation of the gait in the real robot is a promising future work.

\section{ACKNOWLEDGMENTS}

This work was been supported by FCT (Fundação para a Ciência e Tecnologia) in the scope of the project PEstOE/EEI/UI0319/2014.

\section{REFERENCES}

1. L. Hu, C. Zhou, Z. Sun, Biped Gait Optimization Using Spline Function Based Probability Model. Proceedings of the 2006 IEEE International Conference on Robotics and Automation, (2006).

2. F. Faber, S. Behnke, Stochastic optimization of bipedal walking using gyro feedback and phase resetting. Proc. of the IEEE-RAS Int. Conf. on Humanoid Robots (Humanoids) (2007).

3. T. Hemker, M. Stelzer, O. Stryk, H. Sakamoto, Efficient walking speed optimization of a humanoid robot. Int. Journal of Robotics Research, 28 (2): 303-314, (2009).

4. Q. Huang, Z. Yu, W. Zhang, X. Duan, Y. Huang, K. Li, Generation of humanoid walking pattern based on human walking measurement. Proc. of the IEEE-RAS Int. Conf. on Humanoid Robots (Humanoids), (2008).

5. T. Zhe, Z. Changjiu, S. Zengqititle, Humanoid walking gait optimization using GA-based neural network. Advances in Natural Computation, 3611: 252-261 (2005).

6. S. Sakka, K. Yokoi, Humanoid Vertical Jumping based on Force Feedback and Inertial Forces Optimization. Proceedings of the 2005 IEEE International Conference on Robotics and Automation, (2005).

7. "SimTwo", http://www.fe.up.pt/ paco/wiki (2009).

8. R. Smith, Open Dynamics Engine, http://www.ode.org (2000).

9. J. Lima, J. Gonçalves, P. Costa, A. Moreira, Humanoid Robot Gait Planning Resorting to an Adaptive Simulated Annealing Algorithm. Proceedings of 10th Conference on Autonomous Robot Systems and Competitions, (2010).

10. A.I. Pereira, J. Lima, P. Costa, Humanoid Robot Gait Optimization: Stretched Simulated Annealing and Genetic Algorithm a Comparative Study. Numerical Analysis and Applied Mathematics, Volumes I-III (1558) Book Series: AIP Conference Proceedings, pp. 598-601, (2013).

11. S. Fan, M. Sun, Gait Parameters Optimization and Real-Time Trajectory Planning for Humanoid Robots, Advanced Intelligent Computing Theories and Applications. With Aspects of Artificial Intelligence, Lecture Notes in Computer Science Volume 4682, pp. 35-46, (2007).

12. J. Lima, J. Gonçalves, P. Costa, A. Moreira, Humanoid Gait Optimization Resorting to an Improved Simulation Model. International Journal of Advanced Robotic Systems, 10 (67): 1-7 (2013)

13. P. Costa, J. Gonçalves, J. Lima, P. Malheiros, SimTwo Realistic Simulator: A tool for the development and validation of robot software. Theory and Applications of Mathematics and Computer Science, 1 (1): 17-33 (2011).

14. C. Zhou, P.K. Yue, J. Ni, S. Chan, Dynamically Stable Gait Planning for a Humanoid Robot to Climb Sloping Surface. Proceedings of the 2004 IEEE Conference on Robotics, Automation and Mechatronics, (2004).

15. A. Pereira, E.M.G.P. Fernandes, A reduction method for semi-infinite programming by means of a global stochastic approach. Optimization, 58: 713-726 (2009). 